\title{
Differentiability of the value function without interiority assumptions
}

\author{
Juan Pablo Rincón-Zapatero ${ }^{\mathrm{a}, *}$, Manuel S. Santos ${ }^{\mathrm{b}}$ \\ a Departamento de Economía, Universidad Carlos III de Madrid, 28903 Getafe (Madrid), Spain \\ b Department of Economics, University of Miami, Box 248126, Coral Gables, FL 33124, USA
}

\begin{abstract}
This paper studies first-order differentiability properties of the value function in concave dynamic programs. Motivated by economic considerations, we dispense with commonly imposed interiority assumptions. We suppose that the correspondence of feasible choices varies with the vector of state variables, and we allow the optimal solution to belong to the boundary of this correspondence. Under minimal assumptions we prove that the value function is continuously differentiable. We then discuss this result in the context of some economic models, and focus on some examples in which our assumptions are not met and the value function is not differentiable.
\end{abstract}

JEL classification: $\mathrm{C} 61 ; \mathrm{E} 20$

Keywords: Constrained optimization; Value and policy functions; Differentiability; Envelope theorem; Shadow price

\section{Introduction}

Dynamic optimization problems are often analyzed by the methods of dynamic programming which build on properties of the value and policy functions. Although these methods have been extensively studied, there is an important gap that places this methodology really behind the static theory of constrained optimization: General results on the differentiability of the value function have essentially been established for interior optimal solutions. This interiority condition is gen-

\footnotetext{
* Corresponding author

E-mail addresses: jrincon@eco uc3m es (J P Rincón-Zapatero), m santos2@miami edu (M S Santos)
} 
erally subsumed under the following two assumptions: (i) The optimal solution lies in the interior of the choice set (e.g., see [2], and earlier, more limited results of [9] and [13]), and (ii) the choice set does not vary with the vector of states (e.g., see the seminal work of [5], and [12] for further results and economic applications). Both (i) and (ii) turn out to be mathematically equivalent if the constraint correspondence is continuous. For concave optimization the differentiability of the value function can then be established by a well-known static argument in which this function is defined as the envelope of differentiable short-run return functions. This static envelope construction breaks down for boundary solutions if the set of feasible choices varies with the vector of state variables. Indeed, in the absence of (i) and (ii) the derivative of the value function may involve an infinite sum of discounted marginal utilities and returns.

To circumvent the interiority condition we postulate three additional assumptions. First, some optimal choice must lie in the interior of the domain. Second, the matrix of partial derivatives of the saturated constraints must satisfy a full rank condition. The necessity of these two assumptions is well understood from the static theory. The third additional assumption is a new asymptotic condition on the behavior of discounted marginal utilities and returns, and in competitive economies it can be identified with uniqueness of a bubble term over a given equilibrium allocation. We show how our conditions can easily be checked in two specific examples: An extended version of the pure currency model of [10], and an optimal growth model with irreversible investment. Under standard assumptions, in the growth economy the bubble term is always equal to zero, and in the monetary economy the bubble term can only be positive in the case of the Friedman rule or zero nominal interest rate.

We also discuss some other examples in the literature where differentiability fails, linking the lack of differentiability back to the necessary assumptions developed in the paper. Thomas and Worrall [21] study a model of foreign direct investment and find that the value function may not be differentiable for some parameter values. What happens in this model is that the optimal choice may lie in the boundary of the domain. In a related model by Kocherlakota [6] the value function may also display some points of non-differentiability, see [7]. We provide a necessary and sufficient condition for the value function to be differentiable which is tied down to a certain number of constraints being binding. If too many constraints are binding then the constraint qualification is not satisfied. In this model it matters for the dynamics as to whether or not the value function is differentiable. Finally, we discuss a limiting case of an optimal growth model with irreversible investment in which the derivative of the value function becomes unbounded even though the production and utility functions have bounded derivatives.

To prove our differentiability result, we first establish a generalized envelope theorem. The main idea is as follows. For a concave function, a superdifferential always exists at interior points. Suppose further that a full rank condition à la Arrow-Hurwicz-Uzawa (see e.g. [20]) applies as a constraint qualification for the optimal solution. Then, one can use a (generalized) first order condition with respect to tomorrow's choice of the state variable to find values for the Kuhn-Tucker multipliers of the constrained optimization problem at today's state. Our envelope theorem then shows that the superdifferential of the value function can be written in terms of the corresponding multipliers and the superdifferential of the value function at tomorrow's states.

This leads to two formulas for computing the derivative of the value function. First, if eventually (at some finite time $T$ ) the policy falls into the interior of the constraint correspondence, by [2] the derivative of the value function always exists. Then one can iterate backwards to find the derivative of the value function at today's state. A special case of course is the envelope theorem of [2] where $T=0$. Second, if a transversality condition on marginal utilities and returns 
holds, one can iterate forward indefinitely to obtain the value of the derivative. In both cases, the derivative will depend on the Kuhn-Tucker multipliers associated with the saturated constraints.

The importance of these results is clear. First, many problems in macroeconomic theory have relied on differentiability of the value function when the policy function does not fall into the interior of the constraint correspondence. As is well known, results in this literature depend crucially on this assumption (see e.g. Kocherlakota [6]). Hence, having necessary and sufficient conditions for differentiability is important. Second, we show that differentiability of the value function implies that the Kuhn-Tucker multipliers must be unique for concave dynamic programming problems. This entails uniqueness of price systems in decentralized economies or asset pricing models. These shadow values appear as additional state variables in proofs of existence of Markov equilibria for dynamic games of monetary and fiscal policy (see [8] and [15]) and for competitive economies with heterogeneous agents and market frictions [11].

The paper is structured as follows. In Section 2 we set out an abstract (reduced-form) optimization problem, and recall some basic results from dynamic programming. In Section 3 we present our main results on the differentiability of the value function. In Section 4 we consider several economic applications to illustrate the role of our main assumptions. Appendix A contains all the proofs which are not shown in the main text.

\section{The model and preliminary considerations}

We consider an abstract stochastic optimization framework that encompasses various economic applications. For convenience of the presentation, we follow closely the formulation of [19] where the revelation of information is given by an exogenous stochastic process; however, our arguments can readily be modified to account for uncertainty as part of optimization.

\subsection{Stochastic optimization}

Time is discrete, $t=0,1,2, \ldots$. The sequence of choice variables $\left\{x_{t}\right\}_{t} \geqslant 0$ belongs to a set $X \quad \mathbb{R}^{n}$, and the realizations of the exogenous stochastic process $\left\{z_{t}\right\}_{t} \geqslant 0$ lie in a space $Z$. Let $\mathcal{X}$ be the Borel -algebra of $X$ and $\mathcal{Z}$ the -algebra of $Z$. The product space $X \times Z$ is the state space, an is endowed with the product -algebra $\mathcal{X} \times \mathcal{Z}$. We assume that $X$ is convex with non-empty interior.

The primitive elements of our optimization problem are given by a constraint correspondence of feasible choices $\quad: X \times Z \quad 2^{X}$ with graph $=$ Graph( ), a one-period return function $U: \quad \quad \mathbb{R}$, a discount factor $0<\quad<1$, and a transition probability or stochastic kernel $Q: Z \times$ $\mathcal{Z}[0,1]$. We assume that is continuous in $x$ and compact-valued. Moreover, for each $z$ the set $z=\{(x, y): y \quad(x, z)\}$ is convex, and mapping $U(\cdot, \cdot, z)$ is concave and continuous in $(x, y)$. Transition probability $Q$ satisfies the following standard conditions: For each fixed $z \quad Z$ mapping $Q(z, \cdot): \mathcal{Z} \quad \mathbb{R}$ is a probability measure, and for each fixed $B \quad \mathcal{Z}$ mapping $Q(\cdot, B): Z \quad \mathbb{R}$ is a measurable function.

All contingency plans $\left\{x_{t}\right\}_{t} \geqslant 0$ are constructed from the history of past realizations of the process $\left\{z_{t}\right\}_{t} \geqslant 0$ so that every choice vector $x_{t+1}$ available at the end of time $t$ must condition on all the information revealed up to that date. More specifically, let $Z^{t}$ be the space of sequences $z^{t}=\left(z_{1}, z_{2}, \ldots, z_{t}\right)$. For each given $z_{0}$ transition probability $Q$ induces a unique probability measure $\mu^{t}\left(z_{0}, \cdot\right)$ on the product -algebra of $Z^{t}$. A contingency plan $\left\{x_{t}\right\}_{t} \geqslant 0$ is feasible if $x_{t+1}: Z^{t} \quad X$ is a measurable function and $x_{t+1}\left(z^{t}\right) \quad\left(x_{t}\left(z^{t}{ }^{1}\right), z_{t}\right)$, for $z^{t} \quad Z^{t}$ and $t=$ $0,1, \ldots$ 
The stochastic optimization problem can be defined as follows: For each initial condition $\left(x_{0}, z_{0}\right)$, find the maximum value $v\left(x_{0}, z_{0}\right)$ over the set of all feasible contingency plans $\left\{x_{t+1}\left(z^{t}\right)\right\}_{t \geqslant 0}$ for the following discounted infinite-horizon program

$$
v\left(x_{0}, z_{0}\right)=\sup _{\left\{x_{t}\right\}_{t \geqslant 1}}\left\{U\left(x_{0}, x_{1}, z_{0}\right)+\sum_{t=1} t \int_{Z^{t}} U\left(x_{t}, x_{t+1}, z_{t}\right) \mu^{t}\left(z_{0}, d z^{t}\right)\right\} .
$$

We shall often identify optimization problem (1) with the collection of its primitive elements ( ,U, , Q).

\subsection{Value and policy functions}

Let us write Bellman's equation

$$
v(x, z)=\sup _{y}\left\{U(x, y)=\int_{Z} v(y, z) Q(z, d z)\right\} .
$$

As is well known under standard assumptions the value function $v(x, z)$ is measurable, continuous and concave in $x$ for each given $z$, and the unique fixed point of Bellman's equation. Hence, the policy correspondence

$$
H(x, z)=\arg \max _{y}\left\{U(x, z)\{, z)+\int_{Z} v(y, z) Q(z, d z)\right\}
$$

is compact valued. We assume that $H(x, z)$ admits a measurable selection $h(x, z)$. Function $h$ is often referred to as a policy function and defines a time-invariant Markov equilibrium. The existence of a Markov equilibrium can be derived from various standard technical conditions. For the sake of brevity, we refrain discussion of these underlying conditions since they are not needed for our analysis. Note that we allow for multiple stationary Markov equilibrium solutions as function $U(\cdot, \cdot, z)$ is not strictly concave in $(x, y)$. Multiplicity of solutions does not preclude differentiability of the value function.

Consider the Markov operator $\operatorname{M} v(y, z)=\int_{Z} v(y, z) Q(z, d z)$. It follows that for each $z$ function $\operatorname{Mv}(\cdot, z)$ is concave. Hence, at every interior point $y \operatorname{int}(X)$ the superdifferential ${ }_{1}(\mathrm{M} v)(y, z)$ of $\mathrm{M} v(y, z)$ with respect to $y$ is a compact set (e.g., [16]). The following lemma characterizes ${ }_{1}(\mathrm{M} v)$ in terms of ${ }_{1} v$. We need this characterization for our results below.

Lemma 2.1. Let $(, U, \quad, Q)$ be a feasible optimization problem. Then, for every $y \quad \operatorname{int}(X)$ we have

$$
{ }_{1}(\mathrm{M} v)(y, z) \quad 1 \int_{Z} v(y, z) Q(z, d z)=\int_{Z} 1 v(y, z) Q(z, d z) .
$$

This well-established result can be seen as a non-smooth generalization of the differentiation rule under the integral sign. Clarke [4, Theorem 2.7.2, p. 76] offers a version of this result for a Lipschitz function defined on an open set, and hence it must hold for a concave function at an interior point of the domain. The interpretation of (3) is as follows: For $z \quad Z$ fixed, $q_{z}$

${ }_{1}(\mathrm{M} v)(y, z)$ if and only if there is a measurable mapping $z \quad q_{z}(z)$ with $q_{z}(z) \quad{ }_{1} v(y, z)$ almost everywhere (a.e.) in the measure $Q(z, \cdot)$ such that $q_{z}=\int_{Z} q_{z}(z) Q(z, d z)$. 


\section{Differentiability of the value function}

Constrained optimization is pervasive in economics. Constraints may appear in the form of feasibility and technological restrictions, individual rationality and incentive compatibility conditions, transaction costs, borrowing limits, liquidity and collateral requirements, and many other financial frictions. Standard assumptions on utility and production functions do not prevent these constraints from being binding.

\subsection{Main results}

To establish that function $v$ is of class $C^{1}$, we require differentiability of the return function $U$, and some regularity conditions for boundary solutions. We consider two conditions transported from the static theory: A boundary restriction on the policy function over the domain of definition and a constraint qualification (cf. [20]). We also introduce an asymptotic condition on the expected discounted utility of a marginal unit invested today.

D1: For every $x \quad \operatorname{int}(X)$ and $z \quad Z$ function $U(\cdot, \cdot, z)$ is of class $C^{1}$ on some open neighborhood $N(x, y)$ of every point $(x, y)$ with $y \quad H(x, z)$.

If $(x, y)$ belongs to the boundary of $z$ then D1 should be read as that $U(\cdot, \cdot, z)$ admits a differentiable extension on some open neighborhood $N(x, y)$.

D2: For every $x \quad \operatorname{int}(X)$ and $z \quad Z$ there exists $y \quad H(x, z)$ with $y \quad \operatorname{int}(X)$.

This simple assumption says that it is always possible to select an optimal path in the interior of the domain, and should not be confused with the aforementioned interiority condition $y$ $\operatorname{int}(x, z))$. Assumption D2 may be innocuous if the set $X$ can be appropriately redefined, i.e., the domain could be restricted or expanded so that it is not optimal to reach its boundary. We present an example below (Section 4.3) in which D2 does not hold and the value function is not differentiable.

D3: There is a finite collection of functions $g=\left(\ldots, g^{i}, \ldots\right)$, for $i=1,2, \ldots, m$, such that $=\left\{(x, y, z) \quad \mathbb{R}^{n} \times \mathbb{R}^{n} \times Z: g(x, y, z) \geqslant 0\right\}$. Each function $g^{i}(\cdot, \cdot, z): \mathbb{R}^{n} \times \mathbb{R}^{n} \quad \mathbb{R}$ is of class $C^{1}$. Let $I(x, y, z)=\left\{i: g^{i}(x, y, z)=0\right\}$ denote the set of saturated constraints, and let $s(x, y, z)$ denote the cardinality of $I(x, y, z)$. Then for each $(x, z)$ there exists some optimal solution $y \quad H(x, z)$ with $y \quad \operatorname{int}(X)$ such that the rank of the matrix of partial derivatives $\left\{D_{2} g^{i}(x, y, z): i \quad I(x, y, z)\right\}$ is equal to $s(x, y, z)$.

Hence, the graph of the constraint correspondence must be defined by a finite number of constraints. The full rank condition implies that the number of choice variables cannot be less than the number of saturated constraints. The constraint qualification is essential to establish uniqueness of the set of Kuhn-Tucker multipliers as the matrix of partial derivatives $D_{2} g$ can be inverted in a certain generalized sense. If the multipliers are not unique, then in general the superdifferential of the value function will be multivalued (e.g., see Eqs. (13)-(14) below).

We use the following notational conventions. All vectors are column vectors. Then, $D_{j} g(x, y, z)$ for $j=1,2$ is the $m \times n$ matrix of partial derivatives at $(x, y, z)$, and $D_{j} g_{s}(x, y, z)$ for $j=1,2$ refers to the $s \times n$ matrix of partial derivatives of the saturated constraints $\left\{g^{i}(x, y, z): i \quad I(x, y, z)\right\}$. By D3, $D_{2} g_{s}(x, y, z)$ has an $s \times n$ generalized inverse $D_{2} g_{s}^{+}=$ $\left(D_{2} g_{s} D_{2} g_{s}\right){ }^{1} D_{2} g_{s}$ at every $(x, y, z)$, where $D_{2} g_{s}(x, y, z)$ denotes the transpose matrix. In all our computations below, we let the $n \times n$ matrix

$$
G\left(x_{t}, x_{t+1}, z_{t}\right)=D_{1} g_{s}\left(x_{t}, x_{t+1}, z_{t}\right) D_{2} g_{s}^{+}\left(x_{t}, x_{t+1}, z_{t}\right) .
$$


It should be understood that if $x_{t+1} \operatorname{int}\left(\left(x_{t}, z_{t}\right)\right)$ then $G\left(x_{t}, x_{t+1}, z_{t}\right)=\mathbf{0}_{n \times n}$. To simplify notation, let $\mathcal{G}_{t+1}=\prod_{s=0}^{t} G\left(x_{s}, x_{s+1}, z_{s}\right)$. Note that $\mathcal{G}_{1}=G\left(x_{0}, x_{1}, z_{0}\right)$, and $\mathcal{G}_{0}$ will stand for the identity matrix $\mathbf{I}_{n \times n}$.

D4: Let $\left\{x_{t+1}\left(z^{t}\right)\right\}_{t} \geqslant 0$ be an optimal contingency plan with $x_{0} \operatorname{int}(X)$. Then, there is a constant $B_{0}$ such that for every measurable selection of supergradients $q_{t} \quad{ }_{1} v\left(x_{t}, z_{t}\right)$ a.e. in the measure $\mu^{t}\left(z_{0}, \cdot\right)$ we have

$$
\lim t \int_{Z^{t}} \mathcal{G}_{t} q_{t} \mu^{t}\left(z_{0}, d z^{t}\right)=B_{0} .
$$

This transversality condition is only required for boundary solutions. Indeed, $\mathcal{G}_{t}=\mathbf{0}_{n \times n}$ implies that for some history $\left\{z_{s}\right\}_{s=0}^{t}$ every optimal vector $x_{s+1}\left(z^{s}\right)$ belongs to the boundary of $\left(x_{s}, z_{s}\right)$ for all $s=0,1, \ldots, t$. As shown in Proposition 3.2 below for most well-known models we have $B_{0}=0$, and so the above limit is well defined.

In order to prove our main result, we now derive an envelope theorem for constrained, nonsmooth optimization. Let $(x, y, z)=U(x, y, z)+\operatorname{Mv}(y, z)$. Let ${ }_{1,2}$ be the superdifferential of with respect to the first two component variables $(x, y)$. For convex set $z_{0}$ at point $\left(x_{0}, y_{0}\right)$, the normal cone $N_{z_{0}}\left(x_{0}, y_{0}\right)$ is defined as

$$
N_{z_{0}}\left(x_{0}, y_{0}\right)=\left\{\quad \mathbb{R}^{2 n}: \cdot\left(\begin{array}{llll}
x & x_{0}, y & y_{0}
\end{array}\right) \leqslant 0, \quad(x, y) \quad z_{0}\right\} .
$$

Proposition 3.1. Consider a constrained optimization problem ( , $U, \quad, Q)$. Let D1-D3 be satisfied. Then, for any $x_{0} \operatorname{int}(X)$ and $z_{0} \quad Z, q_{0} \quad v\left(x_{0}, z_{0}\right)$ if and only if there exists $q_{1}\left(z_{1}\right) \quad 1 v\left(x_{1}, z_{1}\right)$ a.e. in the measure $Q\left(z_{0}, \cdot\right)$ such that

$$
q_{0}=D_{1} U\left(x_{0}, y_{0}, z_{0}\right)+G\left(x_{0}, y_{0}, z_{0}\right)\left(D_{2} U\left(x_{0}, y_{0}, z_{0}\right)+\int_{Z} q_{1}\left(z_{1}\right) Q\left(z_{0}, d z_{1}\right)\right),
$$

where $y_{0}=h\left(x_{0}, z_{0}\right)$.

Proof. By D1, function $(x, y, z)$ is differentiable with respect to $x$. Hence, $1(x, y, z)=$ $\left\{D_{1}(x, y, z)\right\}$, and so $1,2(x, y, z)=\left\{D_{1} U(x, y, z)\right\} \times 2 \quad(x, y, z)$ for all $(x, y, z)$. Again, by D1-D2 the superdifferential $2(x, y, z)=\left\{D_{2} U(x, y, z)\right\}+{ }_{1}(\mathrm{M} v)(y, z)$. Then, by a standard technical argument (e.g., see Lemma A.1 in Appendix A below), we obtain $q_{0} \quad{ }_{1} v\left(x_{0}, z_{0}\right)$ if and only if there exists $q_{1} \quad{ }_{1}(\mathrm{M} v)\left(y_{0}, z_{0}\right)$ such that

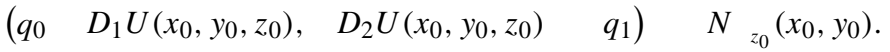

Moreover, as is well known (e.g., [4, Corollary 2, p. 56]) by D3 we must have

$$
\begin{aligned}
& N_{z_{0}}\left(x_{0}, y_{0}\right)
\end{aligned}
$$

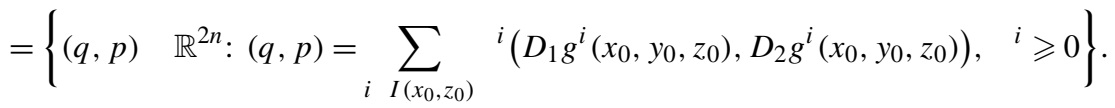

Now, combining this expression with (7) we get

$$
\begin{gathered}
q_{0} D_{1} U\left(x_{0}, y_{0}, z_{0}\right)=\sum_{i}{ }_{I\left(x_{0}, z_{0}\right)}{ }^{i} D_{1} g^{i}\left(x_{0}, y_{0}, z_{0}\right), \\
D_{2} U\left(x_{0}, y_{0}, z_{0}\right) \quad q_{1}=\sum_{i}{ }_{I\left(x_{0}, z_{0}\right)}^{i} D_{2} g^{i}\left(x_{0}, y_{0}, z_{0}\right)
\end{gathered}
$$


for some ${ }^{i} \geqslant 0$, for all $i \quad I\left(x_{0}, z_{0}\right)$. Let $=\left(\ldots,{ }^{i}, \ldots\right)$. Now, from (9) we obtain that $=D_{2} g_{s}^{+}\left(x_{0}, y_{0}, z_{0}\right)\left(D_{2} U\left(x_{0}, y_{0}, z_{0}\right)+q_{1}\right)$. To complete the proof we substitute this expression for into (8) and let $G\left(x_{0}, y_{0}, z_{0}\right)=D_{1} g_{s}\left(x_{0}, y_{0}, z_{0}\right) D_{2} g_{s}^{+}\left(x_{0}, y_{0}, z_{0}\right)$. Finally, recall from Lemma 2.1 that for $z \quad Z$ fixed, $q_{z} \quad{ }_{1}(\mathrm{M} v)(y, z)$ if and only if there is a measurable mapping $z \quad q_{z}(z)$ with $q_{z}(z) \quad{ }_{1} v(y, z)$ a.e. in the measure $Q(z, \cdot)$ such that $q_{z}=\int_{Z} q_{z}(z) Q(z, d z)$.

Proposition 3.1 characterizes the superdifferential of the value function at the current state in terms of the superdifferentials of the instantaneous utility and of the expected value function evaluated at the optimal path. For decentralized economies, this result reduces to the fundamental theorem of finance with binding constraints: The price of an asset is equal to the sum of all expected discounted gross returns where these returns may include the shadow values of the binding constraints.

It is easy to see that a repeated iteration of Lemma 2.1 and Proposition 3.1 yields

$$
\begin{aligned}
q_{0}= & \sum_{t=0}^{T}{ }_{t} \int_{Z^{t}} \mathcal{G}_{t}\left(D_{1} U\left(x_{t}, x_{t+1}, z_{t}\right)+G\left(x_{t}, x_{t+1}, z_{t}\right) D_{2} U\left(x_{t}, x_{t+1}, z_{t}\right)\right) \mu^{t}\left(z_{0}, d z^{t}\right) \\
& +{ }^{T} \int_{Z^{T}} \mathcal{G}_{T} q_{T}\left(z^{T}\right) \mu^{T}\left(z_{0}, d z^{T}\right) .
\end{aligned}
$$

We will show that at every interior point $x_{0}$ the superdifferential ${ }_{1} v\left(x_{0}, z_{0}\right)$ is a singleton, and hence by concavity [16] function $v\left(\cdot, z_{0}\right)$ is differentiable of class $C^{1}$ on $\operatorname{int}(X)$.

Theorem 3.1. Consider a constrained optimization problem ( , $U,, Q)$. Let $\left\{x_{t+1}\left(z^{t}\right)\right\}_{t} \geqslant 0$ be an optimal contingency plan satisfying D1-D4 with $x_{0} \operatorname{int}(X)$. Then, function $v\left(\cdot, z_{0}\right): \operatorname{int}(X) \quad \mathbb{R}$ is differentiable at $x_{0}$ and the partial derivative

$$
\begin{aligned}
D_{1} v\left(x_{0}, z_{0}\right)= & \sum_{t=0} t \int_{Z^{t}} \mathcal{G}_{t}\left(D_{1} U\left(x_{t}, x_{t+1}, z_{t}\right)\right. \\
& \left.+G\left(x_{t}, x_{t+1}, z_{t}\right) D_{2} U\left(x_{t}, x_{t+1}, z_{t}\right)\right) \mu^{t}\left(z_{0}, d z^{t}\right)+B_{0} .
\end{aligned}
$$

Proof. As already pointed out, we just need to show that ${ }_{1} v\left(x_{0}, z_{0}\right)$ is a singleton. By way of contradiction, let $q_{0}, q_{0} \quad{ }_{1} v\left(x_{0}, z_{0}\right)$. Then, after subtracting common terms in (10) there exist $q_{T}\left(z_{T}\right), q_{T}\left(z_{T}\right) \quad{ }_{1} v\left(x_{T}, z_{T}\right)$ for $T \geqslant 0$ such that

$$
q_{0} \quad q_{0}={ }^{t} \int_{Z^{T}} \mathcal{G}_{T}\left(q_{T} \quad q_{T}\right) \mu^{T}\left(z_{0}, d z^{T}\right) .
$$

By assumption D4 this expression converges to zero as $T$ goes to . This proves that ${ }_{1} v\left(x_{0}, z_{0}\right)$ is a singleton at every point $x_{0} \operatorname{int}(X)$. Hence, function $v\left(\cdot, z_{0}\right): \operatorname{int}(X) \quad \mathbb{R}$ is differentiable at $x_{0}$. The value of the derivative $D_{1} v\left(x_{0}, z_{0}\right)$ in (11) is obtained by letting $T$ go to in the above expression (10).

For a given optimal contingency plan $\left\{x_{t+1}\left(z^{t}\right)\right\}_{t \geqslant 0}$, let $E_{T}=\left\{z^{T} \quad Z^{T}: \mathcal{G}_{T+1}=\mathbf{0}_{n \times n}\right\}$. That is, $E_{T}$ is the set of histories at time $T$ such that the optimal solution $\left\{x_{t+1}\left(z^{t}\right)\right\}_{t \geqslant 0}$ lies always in 
the boundary. If with probability one there is a time $T$ such that $E_{T}$ is empty, then D4 trivially holds.

Corollary 3.1. Consider a constrained optimization problem ( ,U, , Q). Let $\left\{x_{t+1}\left(z^{t}\right)\right\}_{t} \geqslant_{0}$ be an optimal contingency plan satisfying D1-D3 with $x_{0} \operatorname{int}(X)$. Assume that there exists a first time $T \geqslant 0$ such that $\mu^{T}\left(z_{0}, E_{T}\right)=0$. Then, function $v\left(\cdot, z_{0}\right): \operatorname{int}(X) \quad \mathbb{R}$ is differentiable at $x_{0}$ and the partial derivative

$$
\begin{aligned}
& D_{1} v\left(x_{0}, z_{0}\right) \\
& =\sum_{t=0}^{T}{ }^{t} \int_{Z^{t}} \mathcal{G}_{t}\left(D_{1} U\left(x_{t}, x_{t+1}, z_{t}\right)+G\left(x_{t}, x_{t+1}, z_{t}\right) D_{2} U\left(x_{t}, x_{t+1}, z_{t}\right)\right) \mu^{t}\left(z_{0}, d z^{t}\right) \\
& \quad+T \int_{Z^{T}} \mathcal{G}_{T} D_{1} U\left(x_{T}, x_{T+1}, z_{T}\right) \mu^{T}\left(z_{0}, d z^{T}\right) .
\end{aligned}
$$

Proof. By the envelope theorem of [2] for $x_{T+1} \operatorname{int}\left(\quad\left(x_{T}, z_{T}\right)\right)$ function $v\left(\cdot, z_{T}\right)$ is differentiable and the derivative $D_{1} v\left(x_{T}, z_{T}\right)=D_{1} U\left(x_{T}, x_{T+1}, z_{T}\right)$. Moreover, $\mathcal{G}_{T+1}=\mathbf{0}_{n \times n}$. Then, expression (12) follows directly from (10). Therefore, the superdifferential $1 v\left(x_{0}, z_{0}\right)$ is unique and $v\left(\cdot, z_{0}\right)$ is differentiable at $x_{0}$.

Of course, for $T=0$ we get the standard envelope theorem $D_{1} v\left(x_{0}, z_{0}\right)=D_{1} U\left(x_{0}, x_{1}, z_{0}\right)$. But it should be noted that for $T>0$ this result requires constraint qualification D3 at those optimal vectors $\left(x_{t}, x_{t+1}, z_{t}\right)$ with $x_{t+1}$ bd $\left.\left(x_{t}, z_{t}\right)\right)$.

\subsection{Duality theory: uniqueness and continuity of the Lagrange multipliers}

As an application of Theorem 3.1 we now show that for every optimal path there exists a unique set of Kuhn-Tucker multipliers satisfying the Euler equations and the transversality condition. By the welfare theorems, the uniqueness of the multipliers entails that an optimal allocation is just supported by a unique price system.

Let $(x, z)$ be a non-negative vector of Kuhn-Tucker multipliers. As shown in the proof of Proposition 3.1, the derivative

$$
D_{1} v(x, z)=D_{1} U(x, h(x, z), z)+D_{1} g(x, h(x, z), z)(x, z)
$$

for every $(x, z)$ such that

$$
D_{2} U(x, y, z)+D_{1} \mathrm{M} v(y, z)+D_{2} g(x, y, z) \quad(x, z)=0 .
$$

Let $\left\{x_{t+1}\left(z^{t}\right)\right\}_{t} \geqslant 0$ be an optimal contingency plan and write $t=\left(x_{t}, z_{t}\right)$. If $v(\cdot, z)$ is differentiable, then by conditions (13) and (14) evaluated over these optimal values we can derive the following system of Euler equations

$$
\begin{aligned}
& D_{2} U\left(x_{t} 1, x_{t}, z_{t} 1\right)+D_{2} g\left(\begin{array}{lllll}
x_{t} & 1 & , x_{t}, z_{t} & 1
\end{array}\right){ }_{t} \quad 1 \\
& +\int_{Z}\left(D_{1} U\left(x_{t}, x_{t+1}, z_{t}\right)+D_{1} g\left(x_{t}, x_{t+1}, z_{t}\right){ }_{t}\right) Q\left(z_{t} 1, d z_{t}\right)=0 \text {, }
\end{aligned}
$$


where $t \geqslant 0$ and $g\left(x_{t}, x_{t+1}, z_{t}\right) \geqslant 0$ with ${ }_{t} g\left(x_{t}, x_{t+1}, z_{t}\right)=0$, for all $t=1,2, \ldots$ To this system of equations we also need to append a transversality condition. For simplicity, let us assume that $X$ is a compact set. Then, let

$$
\lim _{T} \int_{Z^{T}}\left(D_{1} U\left(x_{T}, x_{T+1}, z_{T}\right)+D_{1} g \quad\left(x_{T}, x_{T+1}, z_{T}\right){ }_{T}\right) \mu^{T}\left(z_{0}, d z^{T}\right)=0 .
$$

As is well known (cf. [3]), both (15)-(16) are sufficient conditions ${ }^{1}$ for the characterization of an optimal path $\left\{x_{t}\right\}_{t} \geqslant 0$.

Theorem 3.2. Assume that $X$ is a compact set. Under the conditions of Theorem 3.1, for every optimal contingency plan $\left\{x_{t+1}\left(z^{t}\right)\right\}_{t} \geqslant 0$ with $x_{0} \quad \operatorname{int}(X)$ there exists a unique system of KuhnTucker multipliers $\left\{{ }_{t}\right\}_{t} \geqslant 0$ satisfying (15)-(16).

By (13)-(14) we can construct a system of Kuhn-Tucker multipliers $\left\{t_{t}\right\}_{t} \geqslant 0$ that satisfies the Euler equations (15) and the transversality condition (16). Theorem 3.2 completes the other direction: The system of multipliers $\left\{t_{t}\right\}_{t} \geqslant 0$ satisfying the Euler equations (15) and the transversality condition (16) is unique and corresponds to the derivative of the value function $D_{1} v$ as given by (13)-(14).

In many applications the value function $v(\cdot, \quad)$ varies continuously with a vector of parameters . Then, the derivative of the value function is continuous in , see [16, Ch. 24]. Hence, by Theorem 3.2 the vector of multipliers must also be continuous in .

\subsection{Differentiability and bubbles}

The decomposition given in Theorem 3.1 of the derivative of the value function into the fundamental value and a bubble term is common in asset pricing, where $B_{0}$ can be identified with the bubble term of some existing assets. It is possible to construct examples with a non-null bubble component (e.g. [14] and the cash-in-advance model below), but given that bubbles occur in general equilibrium models under rather pathological circumstances (e.g., [17]), in what follows we will focus on cases in which the bubble term $B_{0}=0$. In our next result we provide some simple conditions that require the value function to be bounded and increasingly monotone.

Proposition 3.2. Consider a constrained optimization problem ( $, U, \quad, Q)$. Assume that $U$ is a bounded function. Let $\left\{x_{t+1}\left(z^{t}\right)\right\}_{t \geqslant 0}$ be an optimal contingency plan with $x_{0} \operatorname{int}(X)$ such that there is a constant $>0$ with $x_{j t} \geqslant$ for each coordinate $j$ and all $t$. Let $G(x, y, z) \geqslant 0$ and $D_{1} U(x, y, z)+G(x, y, z) D_{2} U(x, y, z) \geqslant 0$ for all feasible $(x, y, z)$. Then, under D1-D3 we have $B_{0}=0$ so that expression (4) becomes

$$
\lim _{t} \int_{Z^{t}} \mathcal{G}_{t} q_{t} \mu^{t}\left(z_{0}, d z^{t}\right)=0 .
$$

\footnotetext{
1 The extension of our uniqueness result below to an unbounded domain $X$ requires some further mild regularity conditions. The non-negativity conditions of Proposition 3.2 allow for a simple extension of the transversality condition to unbounded domains, e.g., see Benveniste and Scheinkman [3].
} 
Note that this proposition focuses on non-negative fundamental values in assets in positive supply (cf. [17]). Under some mild regularity assumptions this result can be extended to unbounded return functions. As shown below, the non-negativity conditions are satisfied in standard models of economic growth. If monotonicity does not hold, the bubble term will also vanish in economies in which the fundamental pricing equation can be generated by a contraction mapping in a bounded space of functions (see [9]). In our case, the following conditions ensure this contraction property: (i) Function $U(x, y, z)$ and its partial derivatives $D_{j} U(x, y, z), j=1,2$, are uniformly bounded on $\quad \times Z$, and (ii) $\sup _{(x, y, z)} \quad \int_{Z} G(x, y, z) \mu(z, d z)<1$.

\subsection{Checking our assumptions: two applications}

\subsubsection{A pure currency model}

In the pure currency model of [10] purchases of the aggregate good must be paid for by money held over from the preceding period. At each date the representative agent receives income, $y$, from the sale of goods and government transfers. Current real balances of the household, $m$, and real balances at the beginning of next period, $m$, satisfy $m(1+\quad)+c \quad m \quad y \leqslant 0$, where is an exogenous in ation tax, and $c$ is current consumption. The utility function $u: \mathbb{R}_{+} \times Z \quad \mathbb{R}$ is continuous, where $Z$ is a compact interval of shocks. Moreover, for each $z$ the utility function $u(\cdot, z): \mathbb{R}_{+} \quad \mathbb{R}$ is strictly increasing, concave and of class $C^{1}$.

The recursive formulation of the pure currency model is

$$
\begin{aligned}
& v(m, z)=\max _{c, m \geqslant 0}\left\{u(c, z)+\int_{Z} v(m, z) \mu(d z)\right\} \\
& \text { s.t. } \quad m(1+)+c \quad m \quad y \leqslant 0, \\
& \quad c \quad m \leqslant 0 .
\end{aligned}
$$

For this problem, $U(m, m, z)=u(m+y \quad m(1+\quad), z), \quad(m)=[y /(1+\quad),(y+m) /(1+\quad)]$. Thus, the constraints are $g^{1}(m, m)=m(1+\quad) \quad y \geqslant 0$, and $g^{2}(m, m)=y+m \quad m(1+\quad) \geqslant$ 0 . Let us check conditions D1-D4 in this example. D1 and D2 hold for interior points, $m>0$, and $m>0$ is satisfied along any equilibrium solution of the Lucas' model; D3 is satisfied since $g^{1}, g^{2}$ can never be both active except at $m=0$; D4 is also satisfied for $/(1+)<1$. Indeed, if $g^{1}$ is binding then $G=D_{1} g^{1} / D_{2} g^{1}=0$ since $g^{1}$ is independent of $m$, and if $g^{2}$ is binding then $G=D_{1} g^{2} / D_{2} g^{2}=1 /(1+\quad)$. Therefore, $G<1$ and (4) holds since real balances are bounded below.

Note that the optimal quantity of money or Friedman rule occurs for $/(1+\quad)=1$. In this borderline case, money may simply be a bubble, and we cannot generally show that the limit in D4 is satisfied.

\subsubsection{A simple growth model with irreversible investment}

We provide a simple proof of the differentiability of the value function in the stochastic onesector growth model. We would like to remark that the interiority of optimal solutions is not easy to check in this model, but we will verify that our assumptions are all satisfied. 
Consider the recursive formulation of the problem

$$
\begin{aligned}
& v(x, z)=\max _{c, y \geqslant 0}\left\{u(c, z)+\int_{Z} v(y, z) \mu(z, d z)\right\} \\
& \text { s.t. } \quad c+y=f(x, z), \\
& \quad y \geqslant(1 \quad) x .
\end{aligned}
$$

We assume that functions $u: \mathbb{R}_{+} \times Z \quad \mathbb{R}$ and $f: \mathbb{R}_{+} \times Z \quad \mathbb{R}_{+}$are continuous and $Z$ is a compact set. Moreover, for each $z$ the utility function $u(\cdot, z): \mathbb{R}_{+} \quad \mathbb{R}$ and the production function $f(\cdot, z): \mathbb{R}_{+} \quad \mathbb{R}_{+}$are both increasing, strictly concave and of class $C^{1}$. Let $f(0, z)=$ 0 , and $\lim _{x} \quad 0 \quad D_{1} f(x, z)>1$ and $(1 \quad)+\leqslant \lim _{x} \quad D_{1} f(x, z)<\underline{1}$ for all $z$ and some constant $>0$.

The return function $U: \mathbb{R}_{+} \times \mathbb{R}_{+} \times Z \quad \mathbb{R}$ is defined as $U(x, y, z)=u(f(x, z) \quad y, z)$ and

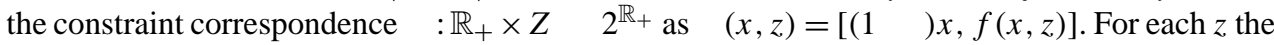
graph of correspondence $(x, z)$ is bordered by two concave functions $g^{1}(x, y, z)=f(x, z)$ $y \geqslant 0$ and $g^{2}(x, y, z)=(1 \quad) x+y \geqslant 0$.

For $x_{0}>0$ assumption D2 trivially holds for an optimal $y$ with $g^{1}(x, y, z)=0$ since $y=$ $f\left(x_{0}, z\right)>0$, and D2 also holds for an optimal $y$ with $g^{2}(x, y, z)=0$ since $y=(1 \quad) x_{0}>0$. The full rank condition in D3 is always satisfied since functions $g^{1}$ and $g^{2}$ are additively separable in $y$. Finally, as it is well known the transversality condition D4 must also hold [17] at every optimal path.

Using contractive arguments, [18] provides a proof of differentiability for boundary solutions in which $g^{2}(x, y, z)=0$. In this case, $G(x, y, z)=(1 \quad)<1$. Our proof allows for optimal solutions to reach the upper boundary $g^{1}(x, y, z)=0$ without imposing a restriction of the type $G(x, y, z)=D_{1} f(x, z)<1$.

\section{Some counterexamples}

\subsection{A simple growth model with irreversible investment}

We show that in the above growth model studied in Section 3.4.2, the derivative of the value function may become unbounded as the stock of capital approaches zero - even if the derivatives of the utility and production functions are bounded. We already discussed that under mild regularity conditions all our assumptions are satisfied and for each $z$ the derivative $D_{1} v\left(x_{0}, z\right)$ exists at all interior points $x_{0}$. The situation is quite different for $x=0$ as $f(0, z)=0$. Then, D2 is not satisfied. Moreover, Fig. 1 depicts a simple deterministic example where D4 does not hold at $x=0$. Here, the derivative $f(0)>1$, and the policy function lies at the upper boundary $h\left(x_{0}\right)=f\left(x_{0}\right)$ for $x_{0}$ near $0 .^{2}$ From Corollary 3.1 for $x_{0}$ near 0 the derivative $v\left(x_{0}\right)=T\left(x_{0}\right) \mathcal{G}_{T\left(x_{0}\right)} v\left(x_{T\left(x_{0}\right)}\right)$ where $T\left(x_{0}\right)$ is the first time $T$ such that $c_{T}>0$ and $\mathcal{G}_{T}=f\left(x_{0}\right) f\left(x_{1}\right) \cdots f\left(x_{T\left(x_{0}\right)}\right)$. Then $T\left(x_{0}\right)$ goes to as $x_{0}$ goes to 0 and ${ }^{T\left(x_{0}\right)} \mathcal{G}_{T\left(x_{0}\right)}=T\left(x_{0}\right) f\left(x_{0}\right) f\left(x_{1}\right) \cdots f\left(x_{T\left(x_{0}\right)}{ }_{1}\right)$ goes to . Consequently, in this example the derivative $v\left(0^{+}\right)$will be unbounded even if the utility and production functions have bounded derivatives.

${ }^{2}$ For $f(0)>\frac{1}{1}$ this optimal policy arises for a linear utility function and for related utility functions sufficiently close to the linear utility. Condition $f(0)>1$ is required for the existence of an interior steady-state solution. 


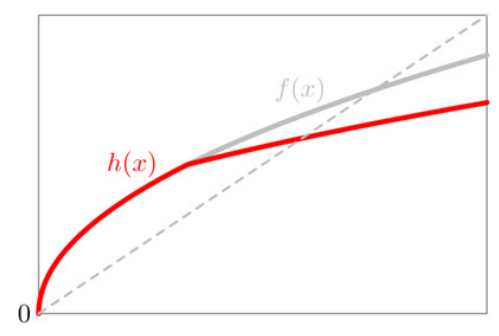

Fig. 1. The derivative of the value function may be unbounded even if the utility and production functions have bounded derivatives.

\subsection{Constrained efficient allocations}

We offer a complete analysis of differentiability of the Pareto frontier for the model of [6]. Koeppl [7] presents an example in which the value function fails to be differentiable. He also provides some sufficient conditions for differentiability. Here we establish a necessary and sufficient condition which is directly linked to the constraint qualification in assumption D3. We thus identify the lack of differentiability of the value function with a failure of assumption D3. Under the special structure of the optimization problem, assumption D4 is not needed. Indeed, in pure exchange economies with no real assets the transversality condition holds trivially.

The recursive formulation of Kocherlakota's exchange economy with two agents is as follows:

$$
\begin{aligned}
& V\left(U_{0}\right)=\max _{\left\{c_{s}, U_{s}\right\}} \sum_{s=1}^{S} s\left[u\left(-c_{s}\right)+V\left(U_{s}\right)\right] \\
& \text { s.t. } \sum_{s=1}^{S} s\left[u\left(c_{s}\right)+U_{s}\right] \geqslant U_{0}, \\
& u\left(c_{s}\right)+U_{s} \geqslant u\left(\begin{array}{c}
1 \\
s
\end{array}\right)+U_{\text {aut }} \text { for all } s,
\end{aligned}
$$

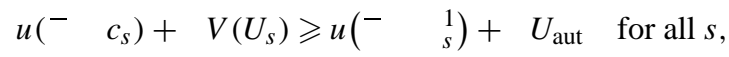

$$
\begin{aligned}
& U_{s} \quad\left[U_{\text {aut }}, U_{\max }\right] \text { for all } s,
\end{aligned}
$$

where the value function $V\left(U_{0}\right)$ assigns the maximum utility to an agent (say agent 2) over all possible utility levels $U_{0}$ of the other agent. Aggregate output is constant, and denoted by ${ }^{-}$, and individual endowments $\quad{ }_{s}>0$, for $i=1,2$, are subject to idiosyncratic shocks, symmetrically distributed, that follow an iid process. The utility function $u$ is bounded, increasing, concave and differentiable.

In the sequel we assume that the Pareto frontier $\left(U_{0}, V\left(U_{0}\right)\right)$ is non-degenerate, that is, $U_{\max }>U_{\text {aut }}$. Let $U_{\max }=V\left(U_{\text {aut }}\right)$. One readily checks that function $V:\left[U_{\text {aut }}, U_{\max }\right] \quad \mathbb{R}$ is well defined, decreasing, concave and continuous. Moreover, even though the value function is in the constraint set, in Appendix A below we extend the above arguments so that our differentiability analysis can be applied.

For given $U_{0}$, let,$\left\{\mu_{s}\right\}$ and $\left\{s_{s}\right\}$ be a set of Kuhn-Tucker multipliers corresponding to constraints $\left(P_{1}\right),\left(P_{2}\right)$ and $\left(P_{3}\right)$, respectively. Let $S_{2}\left(U_{0}\right)$ be the subset of states $s$ where constraint $\left(P_{2}\right)$ is saturated, and $S_{2}^{b}\left(U_{0}\right)$ the subset of states $s$ where constraint $\left(P_{2}\right)$ is binding. That is, $S_{2}\left(U_{0}\right)$ is the subset of states $s$ where constraint $\left(P_{2}\right)$ holds with equality at the optimal solution $\left\{c_{s}, U_{s}\right\}$, and $S_{2}^{b}\left(U_{0}\right)$ is the subset of states $s$ with $\mu_{s}>0$. Analogously, let $S_{3}\left(U_{0}\right)$ be the subset 
of states $s$ where constraint $\left(P_{3}\right)$ is saturated and $S_{3}^{b}\left(U_{0}\right)$ be the subset of states $s$ where constraint $\left(P_{3}\right)$ is binding. Note that $\left(P_{1}\right)$ will always be binding. Also, it is easy to show that the intersection $S_{2}\left(U_{0}\right) \quad S_{3}\left(U_{0}\right)$ is empty (cf. [6]). Hence, for each $s$ there is at most one constraint $\left(P_{2}\right)$ or $\left(P_{3}\right)$ that is saturated.

$$
\mathbf{D 3}^{\prime}: S_{2}\left(U_{0}\right) \quad S_{3}^{b}\left(U_{0}\right)=S \text { and } S_{2}^{b}\left(U_{0}\right) \quad S_{3}\left(U_{0}\right)=S .
$$

Theorem 4.1. Let $U_{0} \quad\left(U_{\text {aut }}, U_{\max }\right)$. Then, $S_{2}\left(U_{0}\right)=S$ and $S_{3}\left(U_{0}\right)=S$. The value function $V$ is differentiable at $U_{0}$ if and only if D3 is satisfied. At points of differentiability, the derivative

$$
V\left(U_{0}\right)=\frac{u\left(^{-} c_{s}\right)}{u\left(c_{s}\right)},
$$

for every state $s$ where none of the constraints $\left(P_{2}\right)-\left(P_{3}\right)$ are binding.

As one can see from the method of proof of Theorem 4.1, assumption D3 is a necessary and sufficient condition. Under this latter condition there is a unique multiplier and so the derivative $V\left(U_{0}\right)=\quad$. Note that D3 can be defined as: (i) $S_{2}\left(U_{0}\right) \quad S_{3}\left(U_{0}\right)=S$ and (ii) for $S_{2}\left(U_{0}\right) \quad S_{3}\left(U_{0}\right)=S$ there must be some non-binding constraint in $S_{2}\left(U_{0}\right)$ and some other nonbinding constraint in $S_{3}\left(U_{0}\right)$. Hence, D3 is slightly weaker than $S_{2}\left(U_{0}\right) \quad S_{3}\left(U_{0}\right)=S$. Condition $S_{2}^{b}\left(U_{0}\right) \quad S_{3}^{b}\left(U_{0}\right)=S$ leaves out some cases in which $V$ is not differentiable, and so this is merely a sufficient condition. By concavity the value function $V$ is differentiable at almost all $U_{0}$. Hence, D3 must be satisfied at almost all $U_{0}$.

\subsection{A model of foreign direct investment}

We brie y discuss a model of foreign direct investment with risk of expropriation by the host country [21]. In this model the value function may fail to be differentiable if D2 is not satisfied.

For illustrative purposes, we focus on the deterministic case. Using Bellman's equation, the optimal contracting problem is written as

$$
\begin{aligned}
& V\left(U_{0}\right)=\max _{I, U}\{I+r(I)+V(U)\} \\
& \text { s.t. } \quad+\quad U \geqslant U_{0}, \\
& r(I)+U \geqslant 0, \\
& V(U) \geqslant 0, \\
& r(I) \geqslant 0, \\
& \geqslant 0 .
\end{aligned}
$$

If none of the constraints are saturated, then optimal investment $I^{*}$ achieves the first-best efficient level, $r\left(I^{*}\right)=1$. Therefore, for first-best Pareto-efficient allocations $(V, U)$ the value function $V$ is differentiable. And since both parties are risk neutral the derivative $V(U)=1$.

Suppose now that a first-best Pareto-efficient allocation cannot be achieved. Then, constraint $\left(R_{2}\right)$ must be binding; moreover, optimality requires that the optimal transfer ${ }_{s}=0$ and so $\left(R_{5}\right)$ is also saturated. Then, by $\left(R_{1}\right)$ the reservation utility $U$ grows over time. As in [21], we consider two cases:

(i) The optimal path eventually reaches an interior, first-best optimal solution. Then, Corollary 3.1 applies as constraint qualification D3 is always satisfied. Therefore, the value function $V$ is differentiable at the initial reservation utility $U_{0}$. 
(ii) The optimal path never reaches an interior, first-best optimal solution. As ${ }_{s}=0$, by $\left(R_{1}\right)$ there is a finite time $T$ such that $V\left(U_{T}\right)=V\left(U_{\max }\right)=0$. Following the method of proof of Proposition 3.1 and (10), one can check that the value function is not differentiable at the smallest point $U_{T} \quad 1$ such that the optimal outcome $U_{\max }=h\left(U_{T}{ }_{1}\right)$. As a matter of fact, we just need to evaluate the directional derivatives of the value function $V$ at $U_{T}{ }_{1}$ from Bellman's equation. To see that these derivatives are different we first note that the optimal policy $U_{1}=h\left(U_{0}\right)$ is increasing. Then, for the right-hand side derivative the term $V\left(U_{T}\right)$ is constant as $V\left(U_{T}\right)=$ $V\left(U_{\max }\right)$, whereas for the left-hand side derivative this term $V\left(U_{T}\right)$ is decreasing with negative slope bounded away from zero. Therefore, both directional derivatives cannot have the same value.

\section{Acknowledgments}

We are indebted to Hector Chade, Adrian Peralta-Alva and Kevin Reffett for some insightful remarks.

\section{Appendix A}

In our first preliminary result we apply basic arguments from convex analysis to the Bellman equation

$$
v(x, z)=\max _{y}\{U(x, z)=\operatorname{Mv} v(y, z)\}
$$

for all $x \quad X$. Recall that $(x, y, z)=U(x, y, z)+\operatorname{Mv}(y, z)$ and 1,2 denotes the superdifferential of with respect to the first two component variables $(x, y)$. In what follows $x_{0}$ refers to an interior point. The normal cone $N_{z_{0}}\left(x_{0}, y_{0}\right)$ of the convex projection set $z_{0}$ at point $\left(x_{0}, y_{0}\right)$ was defined in (5). Throughout the appendix, $y_{0}$ means $y_{0}=h\left(x_{0}, z_{0}\right)$.

Lemma A.1. $q_{0} \quad 1 v\left(x_{0}, z_{0}\right)$ if and only if there exists $(1,2){ }_{1,2}\left(x_{0}, y_{0}, z_{0}\right)$ such that $\left(\begin{array}{lll}q_{0} & 1, & 2\end{array}\right) \quad N_{z_{0}}\left(x_{0}, y_{0}\right)$.

Proof. We follow some well-established arguments, e.g. see [1, Problem 35]. Define the indicator function of set $z$ as

$$
(x, y, z)=\left\{\begin{array}{lll}
0, & (x, y) & z, \\
, & (x, y) / z .
\end{array}\right.
$$

Note that function is concave and upper semicontinuous in $(x, y)$ for $z$ fixed. Now, rewrite Bellman's equation as

$$
v(x, z)=\max _{y}\{(x, y, z)+(x, y, z)\}
$$

for all $x \quad X, z \quad Z$. This is an unconstrained optimization problem. By [1, Prop. 4.3], $q_{0}$ ${ }_{1} v\left(x_{0}, z_{0}\right)$ if and only if $\left(q_{0}, 0\right) \quad{ }_{1,2}(+)\left(x_{0}, y_{0}, z_{0}\right)$. Moreover,

$$
\begin{aligned}
1,2(+)\left(x_{0}, y_{0}, z_{0}\right) & =1,2\left(x_{0}, y_{0}, z_{0}\right)+1,2\left(x_{0}, y_{0}, z_{0}\right) \\
& =1,2\left(x_{0}, y_{0}, z_{0}\right) \quad N_{z_{0}}\left(x_{0}, y_{0}\right) .
\end{aligned}
$$

Therefore, $q_{0} \quad{ }_{1} v\left(x_{0}, z_{0}\right)$ if and only if there exists $(1,2) \quad{ }_{1,2}\left(x_{0}, y_{0}, z_{0}\right)$ such that $\left(q_{0}\right.$ 1, 2) $\quad N_{z_{0}}\left(x_{0}, y_{0}\right)$. 
Proof of Theorem 3.2. Suppose that $\left\{\bar{x}_{t}\right\}_{t \geqslant 0}$ is an optimal contingency plan starting at $\bar{x}_{0}$. For this optimal path assume that there are two sequences of Kuhn-Tucker multipliers $\left\{{ }_{t}\right\}_{t} \geqslant 0$ and $\left\{{ }_{t}\right\}_{t \geqslant 0}$ that satisfy Eqs. (15) and (16). For $z_{0}$ fixed, all initial conditions $x_{0}$, all possible realizations $z_{t}$, and all feasible sequences $x_{t+1} \quad\left(x_{t}, z_{t}\right)$ for $t=1,2, \ldots, n$, let

$$
\begin{aligned}
v_{, n}\left(x_{0}, z_{0}\right)= & \max _{\left\{x_{t}\right\}_{t=1}^{n+1}} \sum_{t=0}^{n} t \int_{Z^{t}} U\left(x_{t}, x_{t+1}, z_{t}\right) \mu^{t}\left(z_{0}, d z^{t}\right) \\
& +{ }^{n+1} \int_{Z^{n+1}} n+1 x_{n+1} \mu^{n+1}\left(z_{0}, d z^{n+1}\right),
\end{aligned}
$$

where ${ }_{n+1}=D_{1} U\left(\bar{x}_{n+1}, \bar{x}_{n+2}, z_{n+1}\right)+D_{1} g\left(\bar{x}_{n+1}, \bar{x}_{n+2}, z_{n+1}\right)_{n+1}$, and

$$
\begin{aligned}
v_{, n}\left(x_{0}, z_{0}\right)= & \max _{\left\{x_{t}\right\}_{t=1}^{n+1}} \sum_{t=0}^{n} t \int_{Z^{t}} U\left(x_{t}, x_{t+1}, z_{t}\right) \mu^{t}\left(z_{0}, d z^{t}\right) \\
& +{ }^{n+1} \int_{Z^{n+1}} n+1
\end{aligned}
$$

where ${ }_{n+1}=D_{1} U\left(\bar{x}_{n+1}, \bar{x}_{n+2}, z_{n+1}\right)+D_{1} g\left(\bar{x}_{n+1}, \bar{x}_{n+2}, z_{n+1}\right)_{n+1}$. Note that the added linear parts $n_{n+1}$ and ${ }_{n+1}$ are chosen so that at point $\bar{x}_{0}$ the optimal solution is $\left\{\bar{x}_{t}\right\}_{t=0}^{n+1}$ for both optimization problems, and for this optimal solution $\left\{t_{t=0}^{n}\right.$ is the sequence of associated KuhnTucker multipliers under (17), and $\left\{{ }_{t}\right\}_{t=0}^{n}$ is the sequence of associated Kuhn-Tucker multipliers under (18). By D3, each sequence of multipliers is unique.

By the same methods as the proof of Theorem 3.1, we can readily see that functions $v, n$ and $v_{, n}$ are concave and of class $C^{1}$ in $x$. Moreover, by (16) and the definitions of ${ }_{n+1}$, ${ }_{n+1}$, the sequences of functions $\left\{v_{, n}\right\}_{n} \geqslant 1$ and $\left\{v_{, n}\right\}_{n} \geqslant 1$ converge uniformly to function $v$. Hence, the sequences of derivative functions $\left\{D_{1} v_{, n}\right\}_{n} \geqslant 1$ and $\left\{D_{1} v,{ }_{n}\right\}_{n} \geqslant 1$ converge uniformly to function $D_{1} v$ on every compact set $K$ int $(X)$ (see [16, Theorem 25.7]). Observe that $D_{1} v{ }_{, n}\left(\bar{x}_{0}, z_{0}\right)=D_{1} U\left(\bar{x}_{0}, \bar{x}_{1}, z_{0}\right)+D_{1} g\left(\bar{x}_{0}, \bar{x}_{1}, z_{0}\right){ }_{0}$, and $D_{1} v{ }_{, n}\left(\bar{x}_{0}, z_{0}\right)=$ $D_{1} U\left(\bar{x}_{0}, \bar{x}_{1}, z_{0}\right)+D_{1} g\left(\bar{x}_{0}, \bar{x}_{1}, z_{0}\right)_{0}$, for all $n$. The convergence of these derivatives to a unique common value implies that $D_{1} g\left(\bar{x}_{0}, \bar{x}_{1}, z_{0}\right)_{0}=D_{1} g\left(\bar{x}_{0}, \bar{x}_{1}, z_{0}\right)_{0}$. Moreover, by the same argument it follows that $D_{1} g\left(\bar{x}_{1}, \bar{x}_{2}, z_{1}\right)_{1}=D_{1} g\left(\bar{x}_{1}, \bar{x}_{2}, z_{1}\right)_{1}$ almost everywhere. Then, by condition D3 applied to (15) we get uniqueness of the multiplier, $0=0$.

Proof of Proposition 3.2. Under the stated non-negativity conditions it is easy to see that at every point $\left(x_{0}, z_{0}\right)$ the superdifferential ${ }_{1} v\left(x_{0}, z_{0}\right)$ must be composed of non-negative vectors. Then this optimization problem can be reconverted into an asset pricing model with real assets along the lines of [17]; see especially their footnote 10. This asset pricing model considers a matrix of returns - which in this case it is given by the vector $D_{1} U(x, y, z)+G(x, y, z) D_{2} U(x, y, z)$ - and a matrix of transformation of securities - which in this case it is given by the matrix $G(x, y, z)$. Hence, interior solutions correspond to one-period assets, and boundary solutions lead to multiperiod assets. The bubble term belongs to long-lived assets starting at $t=0$. Then, for every optimal path $\left\{x_{t+1}\left(z^{t}\right)\right\}_{t \geqslant 0}$ we can generate a sequence of asset prices $q\left(z_{t}\right) \quad{ }_{1} v\left(x_{t}, z_{t}\right)$ so that the asset pricing equation (6) is always satisfied. We can also introduce a single consumption good at each date with relative price equal to unity, and assume that 
the marginal utility of consumption at the optimal point is equal to one. End-of-period asset holdings can be defined in a rather arbitrary way, as the agent can be endowed with new securities at the beginning of each period so as to replicate the optimal path $\left\{x_{t+1}\left(z^{t}\right)\right\}_{t} \geqslant 0$. Hence, under the stated assumptions it follows from [17] that the bubble term $B_{0}=0$.

\section{A.1. An extension of Theorem 3.1 for non-smooth data}

Lemma A.1 remains true if the functions $g^{i}$ are concave but not necessarily smooth. Then, our results can be extended to concave problems with non-smooth data. This extension relies on the characterization of the normal cone to under the much weaker Slater's condition (cf. [16]):

$$
N\left(x_{0}, y_{0}, z_{0}\right)=\left\{(q, p) \quad \mathbb{R}^{2 n}:(q, p) \quad \sum_{i} i_{I\left(x_{0}, z_{0}\right)}^{i}{ }_{1,2} g^{i}\left(x_{0}, y_{0}, z_{0}\right), \quad i \geqslant 0\right\} .
$$

Therefore, our next result will be useful in the model of [6], where the value function appears in the constraints, and may not be differentiable.

For the following result we modify D3 as follows.

$\mathbf{D 3}^{\prime \prime}$ : There is a finite collection of functions $g=\left(\ldots, g^{i}, \ldots\right)$, for $i=1,2, \ldots, m$, such that

$=\left\{(x, y, z) \quad \mathbb{R}^{n} \times \mathbb{R}^{n} \times Z: g(x, y, z) \geqslant 0\right\}$. Each function $g^{i}(\cdot, \cdot, z): \mathbb{R}^{n} \times \mathbb{R}^{n} \quad \mathbb{R}$ is concave.

The following result is a simple consequence of Lemma A.1.

Corollary A.1. Consider a feasible optimization problem ( , U, , Q). Let D1-D3 be satisfied. For any $x_{0} \quad \operatorname{int}(X)$ and $z_{0} \quad Z$, we must have: $q_{0} \quad{ }_{1} v\left(x_{0}, z_{0}\right)$ if and only if there exists $\left(\begin{array}{ll}1,2 & 2\end{array} \quad\left(x_{0}, y_{0}, z_{0}\right)\right.$ such that $\left(q_{0} \quad 1, \quad 2\right) \quad N_{z_{0}}\left(x_{0}, y_{0}\right)$ for every $y_{0} \quad \operatorname{int}(X)$.

Proof of Theorem 4.1. First we show that $S_{2}\left(U_{0}\right)=S$ and $S_{3}\left(U_{0}\right)=S$. Suppose not. For concreteness, assume that $S_{2}\left(U_{0}\right)=S$. Then, summing over $\left(P_{2}\right)$ we get that $U_{0}=\sum_{s=1}^{S} \quad{ }_{s}\left[u\left(c_{s}\right)+\right.$ $\left.U_{s}\right]=U_{\text {aut }}$. But this is in contradiction with $\left(P_{1}\right)$ as $U_{0}>U_{\text {aut }}$.

By Corollary A.1 and the characterization of $N\left(x_{0}, y_{0}, z_{0}\right)$ in (19), we now have to prove that the superdifferential of $V$ at $U_{0}$ is unique. As this is an exchange economy, one should expect the derivative of the value function to depend on current utilities. Indeed, following Kocherlakota [6] for any $U_{0} \quad\left(U_{\text {aut }}, U_{\max }\right)$, we get the following system of first-order conditions

$$
\begin{aligned}
& 0={ }_{s} u\left({ }^{-} c_{s}\right)+{ }_{s} u\left(c_{s}\right)+\mu_{s} u\left(c_{s}\right) \quad{ }_{s} u\left(\left(^{-} c_{s}\right),\right. \\
& 0={ }_{s} q_{s}+{ }_{s}+\mu_{s}+{ }_{s} q_{s},
\end{aligned}
$$

for some $q_{s} \quad V\left(U_{s}\right)$ for all $s$, and,$\left\{\mu_{s}\right\}_{s}$, and $\{s\}_{s}$ are Kuhn-Tucker multipliers corresponding to the constraints $\left(P_{1}\right),\left(P_{2}\right)$, and $\left(P_{3}\right)$, respectively. It turns out that the first equation $(20)$ suffices to pin down the multiplier , and hence the derivative of the value function depends on the consumption allocation in the first period.

To study the solutions of system (20)-(21) we distinguish four cases that encompass all possibilities. In the first two cases we consider that $q_{s}=q_{s}$ in (21). This does not entail any loss of generality, since the case $q_{s}=q_{s}$ may add other multiple solutions.

(i) $S_{2}^{b}\left(U_{0}\right) \quad S_{3}^{b}\left(U_{0}\right)=S$. That is, for every $s$ we have that either $\mu_{s}>0$ or ${ }_{s}>0$. Note that $>0$. Assume that $q_{s}=q_{s}$ in (21). Multiplying (21) by $\quad \underline{u\left(c_{s}\right)}$, and adding up (20) and (21) we get that $q_{s}=\frac{u\left({ }^{-} c_{s}\right)}{u\left(c_{s}\right)}$. Hence, in this case (20) and (21) are always collinear. Therefore, to see 
the determinacy of these multipliers it suffices to consider the system of Eq. (20). There are then $S$ equations in $S+1$ unknowns,,$\mu_{s}$ and ${ }_{s}$. (Note that $\mu_{s}$ and ${ }_{s}$ appear only in the equation associated with state $s$ and either $\mu_{s}>0$ or ${ }_{s}>0$.) It follows that there are multiple solutions, and so there is a continuum of that satisfy (20) and (21).

(ii) $S_{2}^{b}\left(U_{0}\right) \quad S_{3}\left(U_{0}\right)=S$ or $S_{2}\left(U_{0}\right) \quad S_{3}^{b}\left(U_{0}\right)=S$, but $S_{2}^{b}\left(U_{0}\right) \quad S_{3}^{b}\left(U_{0}\right)=S$. For simplicity, let us just consider $S_{2}^{b}\left(U_{0}\right) \quad S_{3}\left(U_{0}\right)=S$, where $\mu_{s}>0$ for all $s \quad S_{2}^{b}\left(U_{0}\right)$ and $s \geqslant 0$ for all $s \quad S_{3}\left(U_{0}\right)$ with $\quad s=0$ for some $s$. As in (i), indeterminacy of the solutions does exist, but the multiplier can only be increased from the original value as some ${ }_{s}=0$. (If is decreased then some ${ }_{s}$ is forced to be a negative number.)

(iii) $S_{2}^{b}\left(U_{0}\right) \quad S_{3}\left(U_{0}\right)=S$ and $S_{2}\left(U_{0}\right) \quad S_{3}^{b}\left(U_{0}\right)=S$ but $S_{2}\left(U_{0}\right) \quad S_{3}\left(U_{0}\right)=S$. In this case, the multiplier is unique, and it is just determined by (20). It cannot be increased because in the above equation system (20) there is some $\mu_{s}=0$, and it cannot be decreased because there is some ${ }_{s}=0$.

(iv) $S_{2}\left(U_{0}\right) \quad S_{3}\left(U_{0}\right)=S$. In this case there is some state $s$ for which neither $\left(P_{2}\right)$ nor $\left(P_{3}\right)$ are saturated. Then, for some $s$ we have that both $\mu_{s}$ and $s$ are equal to zero. It then follows from (20) that is unique.

Therefore, in cases (iii)-(iv) the multiplier is unique and the value of the derivative $V\left(U_{0}\right)=$ is as stated in the theorem.

\section{References}

[1] J.P. Aubin, Optima and Equilibria - An Introduction to Nonlinear Analysis, Springer-Verlag, New York, 1993.

[2] L.M. Benveniste, J.A. Scheinkman, On the differentiability of the value function in dynamic models of economics, Econometrica 47 (1979) 727-732.

[3] L.M. Benveniste, J.A. Scheinkman, Duality theory for dynamic optimization models of economics: The continuous time case, J. Econ. Theory 27 (1982) 1-19.

[4] F.H. Clarke, Optimization and Nonsmooth Analysis, SIAM, New York, 1990.

[5] J.M. Danskin, The Theory of Maxmin, Springer-Verlag, New York, 1967.

[6] N.R. Kocherlakota, Implications of efficient risk sharing without commitment, Rev. Econ. Stud. 63 (1996) 595-609.

[7] T.V. Koeppl, Differentiability of the efficient frontier when commitment to risk sharing is limited, Top. Macroecon. 6 (1) (2006), article 10, http://www.bepress.com/bejm/topics/vol6/iss 1/art10.

[8] F.E. Kydland, E.C. Prescott, Dynamic optimal taxation, rational expectations and optimal control, J. Econ. Dynam. Control 2 (1980) 79-91.

[9] R.E. Lucas, Asset prices in an exchange economy, Econometrica 46 (1978) 1426-1445.

[10] R.E. Lucas, Equilibrium in a pure currency economy, Econ. Inquiry 18 (1980) 203-220.

[11] J. Miao, M.S. Santos, Existence and computation of competitive equilibria in dynamic non-optimal economies, manuscript, Boston University.

[12] P. Milgrom, I. Segal, Envelope theorems for arbitrary choice sets, Econometrica 70 (2002) 583-601.

[13] P. Milgrom, I. Zilcha, On optimal growth under uncertainty, J. Econ. Theory 11 (1975) 329-339.

[14] L. Montrucchio, F. Privileggi, On fragility of bubbles in equilibrium asset pricing models of Lucas-type, J. Econ. Theory 101 (2001) 158-188.

[15] C. Phelan, E. Stacchetti, Sequential equilibria in a Ramsey tax model, Econometrica 69 (2001) 1491-1518.

[16] R.T. Rockafellar, Convex Analysis, Princeton Univ. Press, Princeton, NJ, 1970.

[17] M.S. Santos, M. Woodford, Rational asset pricing bubbles, Econometrica 65 (1997) 19-57.

[18] T.J. Sargent, Tobin's $q$ and the rate of investment in general equilibrium, in: K. Brunner, A. Meltzer (Eds.), On the State of Macroeconomics, in: Carnegie-Rochester Conf. Ser., vol. 12, Elsevier, Amsterdam, 1980, pp. 107-154.

[19] N.L. Stokey, R.E. Lucas, E.C. Prescott, Recursive Methods in Economic Dynamics, Harvard Univ. Press, Cambridge, MA, 1989.

[20] A. Takayama, Mathematical Economics, Cambridge Univ. Press, Cambridge, MA, 1990.

[21] J. Thomas, T. Worrall, Foreign direct investment and the risk of expropriation, Rev. Econ. Stud. 61 (1994) 81-108. 